\title{
A patient's temperature remote control system based on NODEMCU ESP8266
}

\author{
Anas El Kasmi ${ }^{1}$, Mostafa Abouricha ${ }^{2}$, and Abdelkader Boulezhar ${ }^{3}$ \\ ${ }^{1}$ LRESD, Department of Physics, Faculty of Sciences-AÏn Chock, Hassan II University of Casablanca, BP 20100, Morocco \\ ${ }^{2}$ LMTI, Department of Physics, Faculty of Sciences Ibn Zohr University of Agadir BP 8106, Morocco. \\ ${ }^{3}$ LRESD, Department of Physics, Faculty of Sciences-AÏn Chock, Hassan II University of Casablanca, BP 20100, Morocco
}

\begin{abstract}
With the prompt development in wireless communication, in addition to the evolution of wireless patient monitoring system, and to reduce the death of a big number of patients who endear as a result of heart failure or sudden high temperature in children. This paper proposed a sophisticated method of observation human body parameters like temperature measurements with employ at NodeMcu ESP8266 unit in the Internet of Things (IoT). This prototype is based on NodeMcu module (a static access point that provides the WIFI network, a server, a client and a mobile access point attached to the remote surveillance object) programmed under Arduino IDE and communicating between them via the HTTP protocol.
\end{abstract}

\section{INTRODUCTION}

The development of the medical field largely resulted from the rapid development of technology. This has led to an increase in the average age of human beings and low mortality rates, especially in industrialized and developed countries [1]. The lack of modern medical equipment that helps diagnose and treat patients has led to poor medical services in developing countries and weak technology such as Third World countries .

Today, due to advances in technology and the Internet of Things, step-by-step tasks such as mailing, accessing bank records, monitoring the degree of other tasks [1], eHealth [2], smart home [3-4], smart city [5], intelligent transport [6], wireless sensor networks [7], smart grid [8], objects of remote monitoring systems [9]. However for any IoT application[10].

Here in this project, we will create an IoT based health monitoring system that records body temperature and also send an email alert. Readings are recorded in the ThingSpeak and Google Sheets so that the patient's health can be monitored from anywhere in the world via the Internet.

Note that the communication between the different nodes is done by the HTTP protocol of the library ESP8266.h and the integrated development environment Arduino IDE.

The rest of the paper is organized as follows. In section 2 related works were defined. In section 3 proposed system were explained.. In section 4 Hardware realizations were shown. In section 5 System implementation and the conclusion and future work was discussed in section 6 .

\section{NODEMCU (ESP8266)}

The NodeMcu ESP8266 includes a WiFi module, memory, a serial link and manages GPIO ports. This microcontroller has the ability to perform activities related to WIFI.

In this article, we will discuss the use of the NodeMcu ESP8266 unit in the Internet of Things, (IoT) using AT or Arduino IDE commands for data exchange.

$-802.11 \mathrm{~b} / \mathrm{g} / \mathrm{n}$ wireless standard

-Support three STA / AP / STA + AP working modes

-Built-in TCP / IP protocol stack to support multiple TCP client connections (5 MAX)

-AD0: 1 ADC channel

-Power input: 4.5V - 9V (10VMAX), powered by USB \

-Transfer rate: $110-460800 \mathrm{bps}$

-Support UART / GPIO data communication interface

-Remote Firmware Upgrade (OTA:Over-the-air programming)

-Smart Link Smart Grid Support \ 


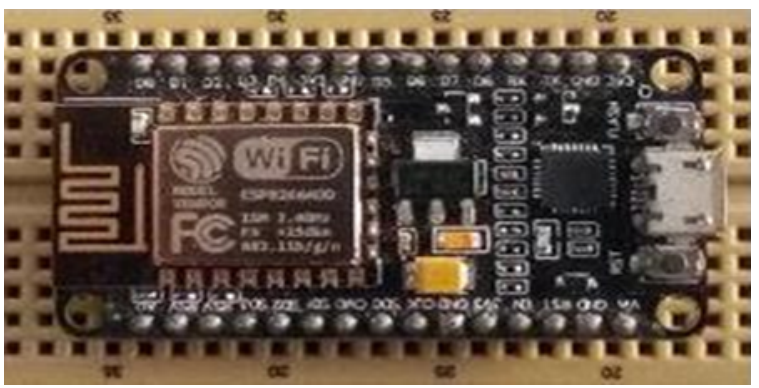

Fig. 1. The image and main characteristics of the NodeMcu used

\section{Pinout and connectivity :}

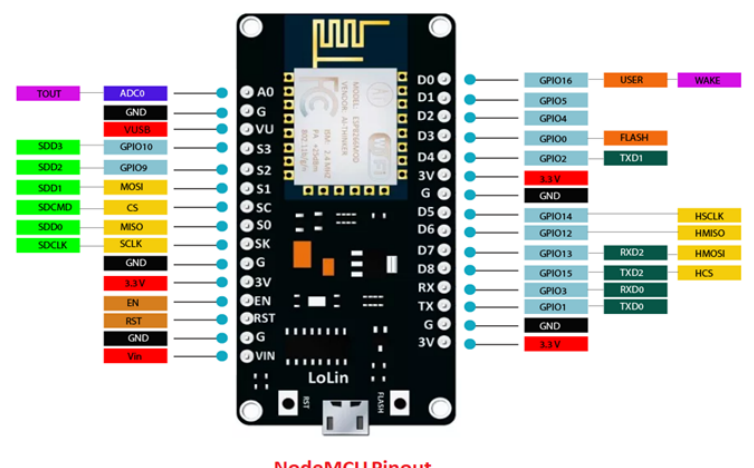

Fig. 2. Pinout and connections of the NodeMcu card

\subsection{LM35 temperature sensor}

This component makes it possible to measure the temperature around the sensor. It is precise, inexpensive, very easy to use and extremely reliable. The temperature measured by the sensor is proportional to the voltage on the analog output of the sensor. To reduce the difference between the actual temperature and the measured temperature, especially at extremes (around $-55^{\circ}$ and around $150^{\circ}$ ).

\subsubsection{Features}

- Calibrated Directly in Celsius (Centigrade)

- Linear + $10-\mathrm{mV} /{ }^{\circ} \mathrm{C}$ Scale Factor

- $0.5^{\circ} \mathrm{C}$ Ensured Accuracy (at $25^{\circ} \mathrm{C}$ )

- Rated for Full $-55^{\circ} \mathrm{C}$ to $150^{\circ} \mathrm{C}$ Range

- Suitable for Remote Applications

- Low-Cost Due to Wafer-Level Trimming

- Operates From $4 \mathrm{~V}$ to $30 \mathrm{~V}$

- Less Than 60- $\mu \mathrm{A}$ Current Drain

- Low Self-Heating, $0.08^{\circ} \mathrm{C}$ in Still Air

- Non-Linearity Only $\pm 1 / 4{ }^{\circ} \mathrm{C}$ Typical

- Low-Impedance Output, $0.1 \Omega$ for 1-mA Load

\subsubsection{Applicaions}

Power Supplies

- Battery Management

- HVAC
- Appliances

\subsubsection{Description}

The LM35 series are precision integrated-circuit temperature devices with an output voltage linearly proportional to the Centigrade temperature. The LM35 device has an advantage over linear temperature sensors calibrated in Kelvin, as the user is not required to subtract a large constant voltage from the output to obtain convenient Centigrade scaling. The LM35 device does not require any external calibration or trimming to provide typical accuracies of $\pm 1 / 4^{\circ} \mathrm{C}$ at room temperature and $\pm 3 / 4^{\circ} \mathrm{C}$ over a full $-55^{\circ} \mathrm{C}$ to $150^{\circ} \mathrm{C}$ temperature range. Lower cost is assured by trimming and calibration at the wafer level. The lowoutput impedance, linear output, and precise inherent calibration of the LM35 device makes interfacing to readout or control circuitry especially easy. The device is used with single power supplies, or with plus and minus supplies. As the LM35 device draws only $60 \mu \mathrm{A}$ from the supply, it has very low self-heating of less than $0.1^{\circ} \mathrm{C}$ in still air. The LM35 device is rated to operate over a $-55^{\circ} \mathrm{C}$ to $150^{\circ} \mathrm{C}$ temperature range, while the $\mathrm{LM} 35 \mathrm{C}$ device is rated for a $-40^{\circ} \mathrm{C}$ to $110^{\circ} \mathrm{C}$ range $\left(-10^{\circ}\right.$ with improved accuracy). The LM35series devices are available packaged in hermetic TO transistor packages, while the LM35C, LM35CA, and LM35D devices are available in the plastic TO-92 transistor package. The LM35D device is available in an 8-lead surface-mount small-outline package and a plastic TO-220 package.

\subsection{ThingSpeak}

ThingSpeak provides a great tool for IoT based projects. With ThingSpeak, we can monitor our data and control an online system, using the web pages provided by ThingSpeak, we can collect sensor data, analyze it, visualize it and finally trigger actions.

ThingSpeak will be used to monitor temperature online over the Internet. For this, it is necessary to set up the IFTTT platform to connect ThingSpeak to the messaging service so that the alert message can be sent whenever the patient is in a critical state.

1st step: Creation of a user account on ThingSpeak.com.

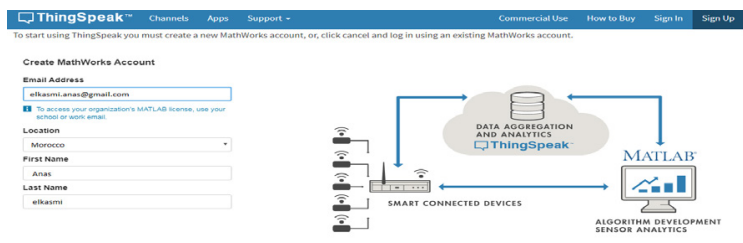

Fig. 3. Window to create a count on Thingspeak.com site 
2nd step: In the "Channels" menu and click on the New channel option on the same page to create a new channel.

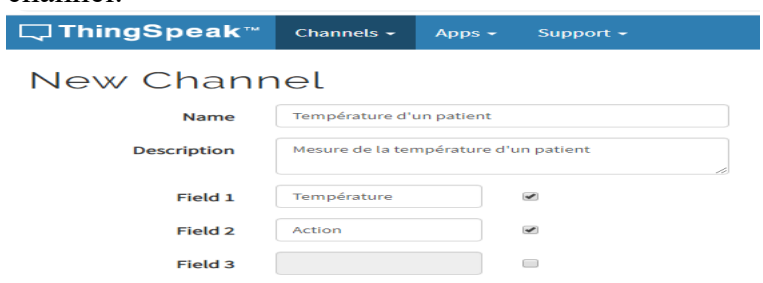

Fig. 4. Window to create channel and fields on Thingspeak.com site

3rd Step: Now you will see a template for channel creation, fill in the name and description as you want. Fill in "Temperature" and "Action" in the fields Field 1 , Field 2, check the boxes of the fields. Finally, check Save channel. Now your new channel has been created

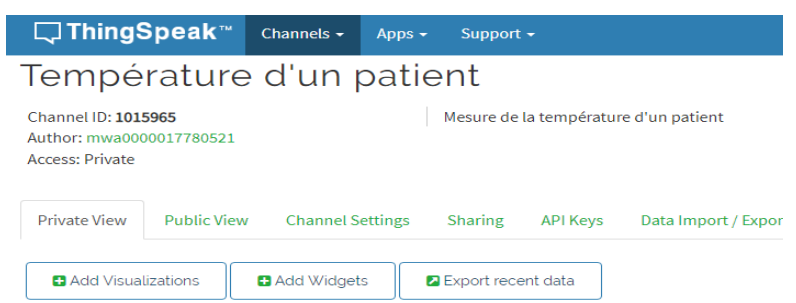

Fig. 5. Window's name of channel and action to generate

4th Step: You will see three graphics as shown below. Note the write API key and we'll use that key in our code.

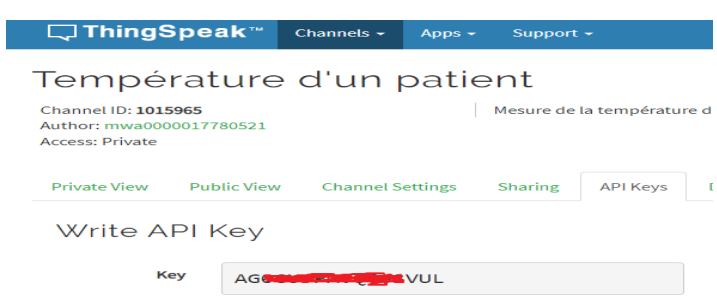

Fig. 6. Window to show write API key on the channel

5th Step: Now we will use the ThingHTTP app on the server to launch the IFTTT app to enter data into Google Sheets and send emails / SMS

\subsection{ThingHTTP and IFTTT}

ThingHTTP enables communication between devices, websites, and web services without having to implement the protocol at the device level. You can specify actions in ThingHTTP, which you want to perform with other ThingSpeak apps such as React. To make ThingHTTP new, we'll need a URL to run which we'll get from IFTTT

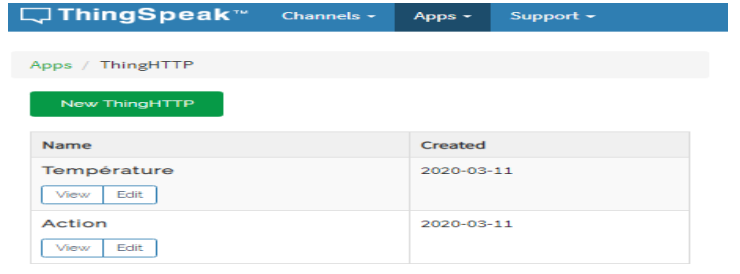

Fig. 8. Window showing communications between ThingHTTP and IFTTT app

\subsection{IFTTT:}

IFTTT is a separate business from Google. Its name means "If This Then That".Your custom command and action form a whole called an "applet".

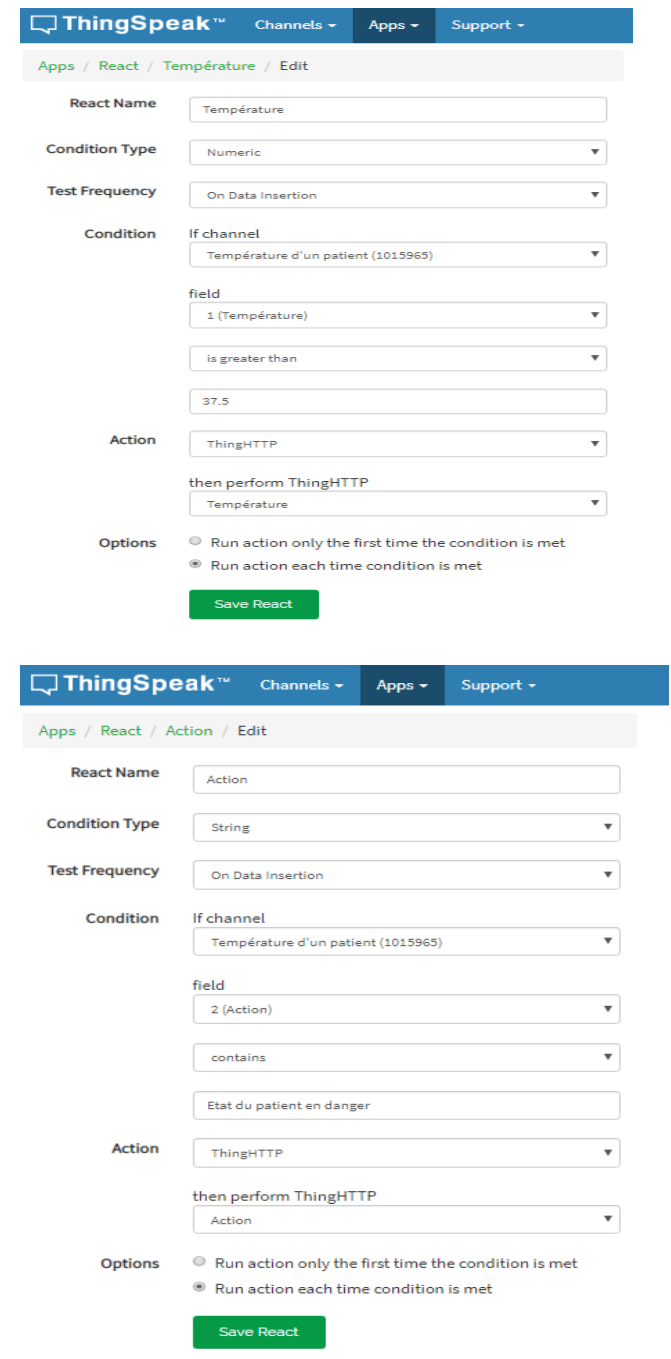

Fig. 9. Windows of IFTTT applets

\section{PROPOSED SYSTEM}

The LM35DZ temperature sensor is powered by $3.3 \mathrm{v}$ generated by the NodeMcu board, the analog value Vin image of the temperature is sent to the Node board for processing. 
The transfer of data between the NodeMcu card and the computer must go through the ThingSpeak site which is a website for the IoT. It is an interface that is used to connect software to services, to collect, store and analyze data.

\section{HARWARD REALIZATION}

In The Figure 10 shows the components of an "object detection and monitoring" system that includes: The LM35DZ temperature sensor, an NodeMcu.

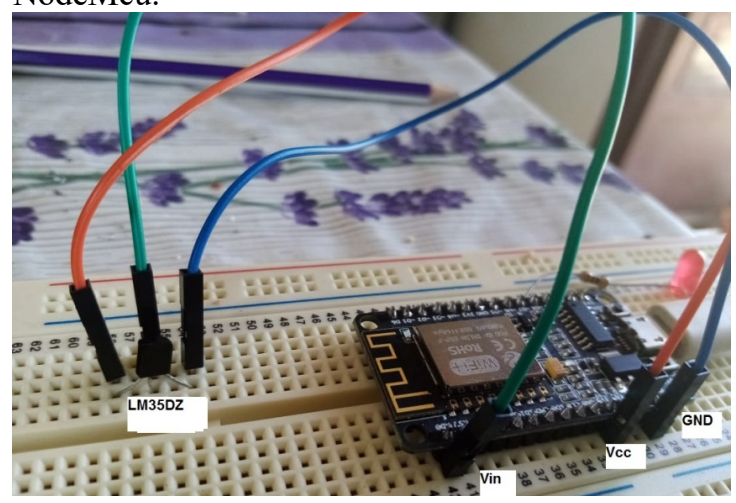

Fig. 10. Components of the remote monitoring system of an object in real time

\subsection{Software Part (Code)}

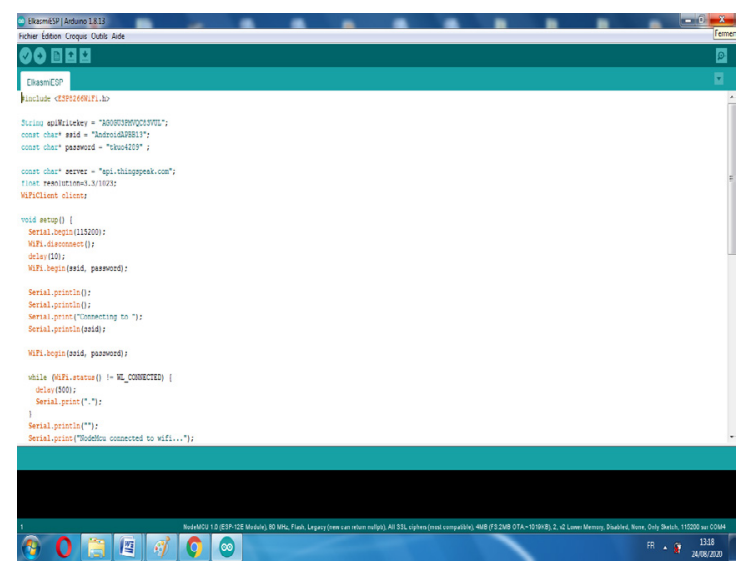

Fig. 11. main program code with arduino IDE

\subsection{System Implementation}

The operation of the measuring system is based on the developed program which is executed by the NodeMcu card, which will then deliver all the measurements obtained each determined time interval.

The next is to send the measurements taken via wifi to the ThingSpeak site to be saved and displayed as graphs. The flowchart for the transmission of data from the NodeMcu card to the computer is shown in the figure.

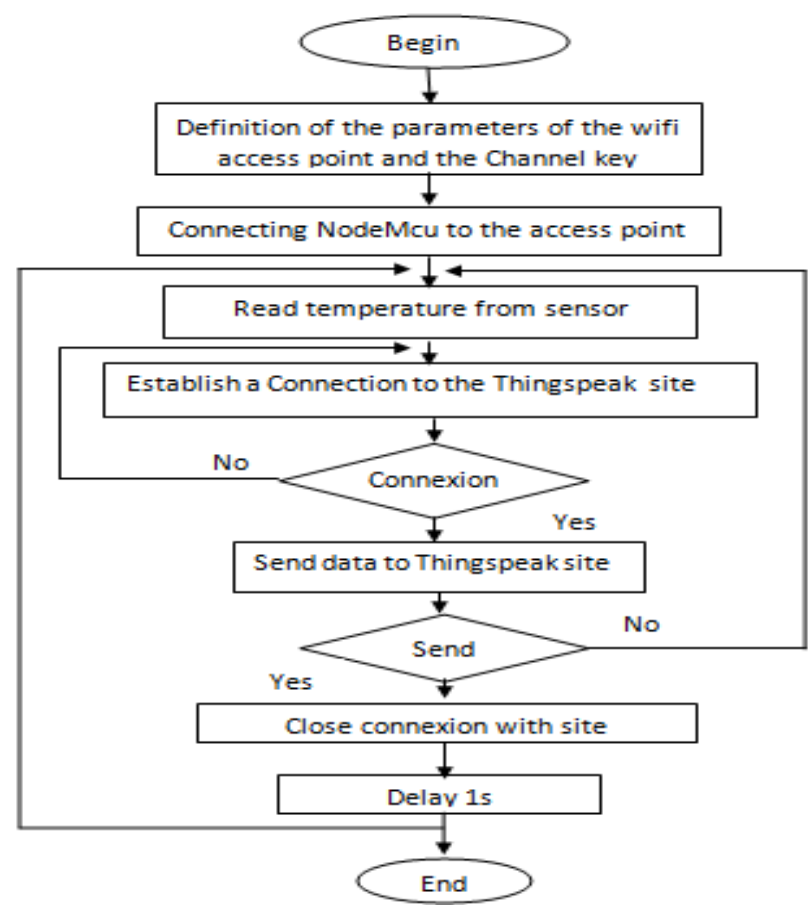

Fig. 12. flowchart for the transmission of data.

\section{RESULTS AND DISCUSSIONS}

\subsection{Temperature Measurement}

When turn on the energy, the system consists in sending the measurements carried out via wifi to the ThingSpeak site to be saved and displayed as graphs.

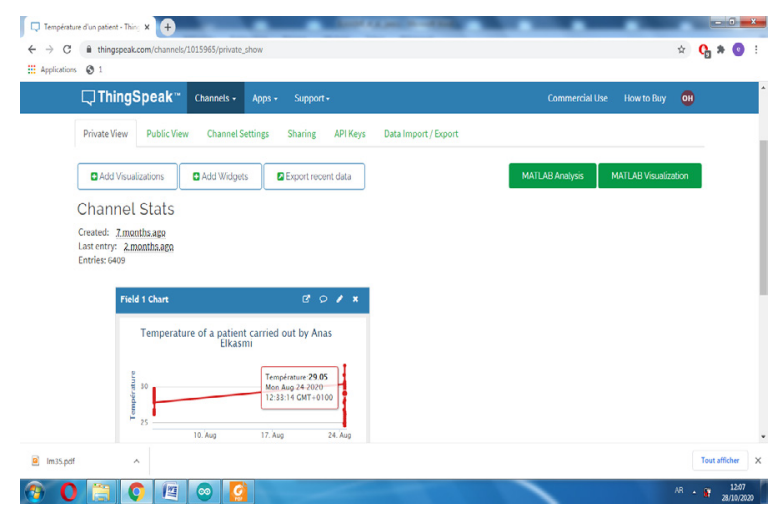

Fig. 13. Window of channel state on thingspeak.com site at the beginning of measurements 


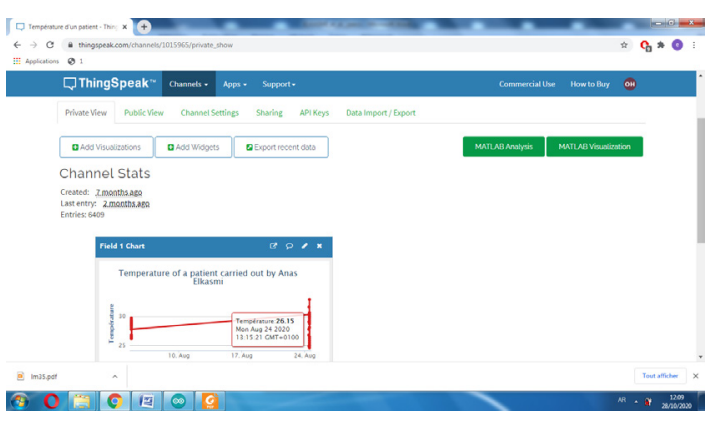

Fig. 14. Window of channel state on thingspeak.com site at other times

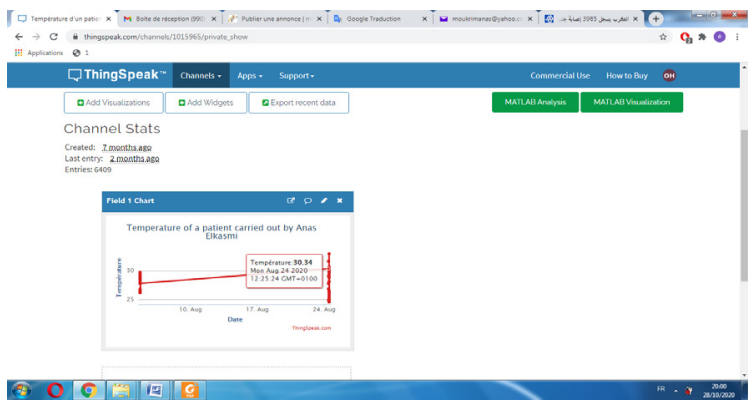

Fig. 15. Window of channel state on thingspeak.com site at other times

\subsection{Discussions}

The first series of tests with the NodeMCU assembled with Thingspeak server allowed us to obtain the following curve: the delay for taking measurements is carried out regularly by setting the "Delay" loop from the Arduino code one second for our example.

\section{CONCLUSION}

In this work we sought to design a system for measuring the temperature of a patient. It is composed of a temperature sensor, a NodeMcu microcontroller card whose the role is data processing and sending measurements to the ThingSpeak site for be recorded and displayed as graphs on a computer. As perspectives of this work, extensions of the measurement system can be considered taking into account other health variables such as blood pressure measurement and other measurements.

\section{ACKNOWLEDGEMENTS}

The authors are very much thankful to the unanimous reviewers of the paper and editors of the journal for their constructive and helpful comments that improved the quality of the paper.

\section{REFERENCES}

1. E. Teaw, et al., "A Wireless Health Monitoring System," International Conference on Information Acquisition, 2005, June 27- July 3.

2. P. Gupta et al., "An IoT Framework for Addressing Parents Concerns about Safety of School Going Children," International Journal of Electrical and computer Engineering (IJECE), vol. 6, $\mathrm{n}^{\circ} 6$, pp. 3052, 2016.

3. N. A. A. Rahman, et al., "Biomedical health monitoring system design and analysis," Indonesian Journal of Electrical Engineering and Computer Science, Vol 13, pp. 1056-1064, 2019.

4. B. Li, et al., "Research and Application on the Smart Home Based on Component Technologies and Internet of Things," Procedia Engineering, Vol 15, pp. 2087-2092, 2011.

5. S. Kumar, et al., "Ubiquitous Smart Home System Using Android Application," International journal of Computer Networks \& Communications, vol. 6, no 1, pp. 33-43, janv. 2014.

6. V. A. Memos, et al., " An Efficient Algorithm for Mediabased Surveillance System (EAMSuS) in IoT Smart City Framework," Future Generation Computer Systems, vol. 83, pp. 619-628, 2018.

7. P. S. Saarika, et al., " Smart transportation system using IoT," 2017 International Conference On Smart Technologies For Smart Nation (SmartTechCon), Bangalore, pp. 1104-1107, 2017.

8. V. Suryani,, et al., " ConTrust: A Trust Model to Enhance the Privacy in Internet of Things," International Journal of Intelligent Engineering and Systems, $\operatorname{Vol} 10 n^{\circ} 3$, pp. 30-37, 2017.

9. R. Habachi, et al., " Resolution of economic dispatch problem of the morocco network using crow search algorithm," Indonesian Journal of Electrical Engineering and Computer Science, vol. 13, n¹, pp. 347, janv. 2019.

10. K. Radharamana, et al., "Web Architecture for Monitoring Field using Representational State Transfer Methods," International Journal of Intelligent Engineering and Systems, vol. 12, pp. 84-93, 2019.

11. P. Asghari, et al., " Internet of Things applications: A systematic review," Computer Networks, Vols 148, pp. 241261, janv 2019 


\section{BIOGRAPHIES OF AUTHORS}

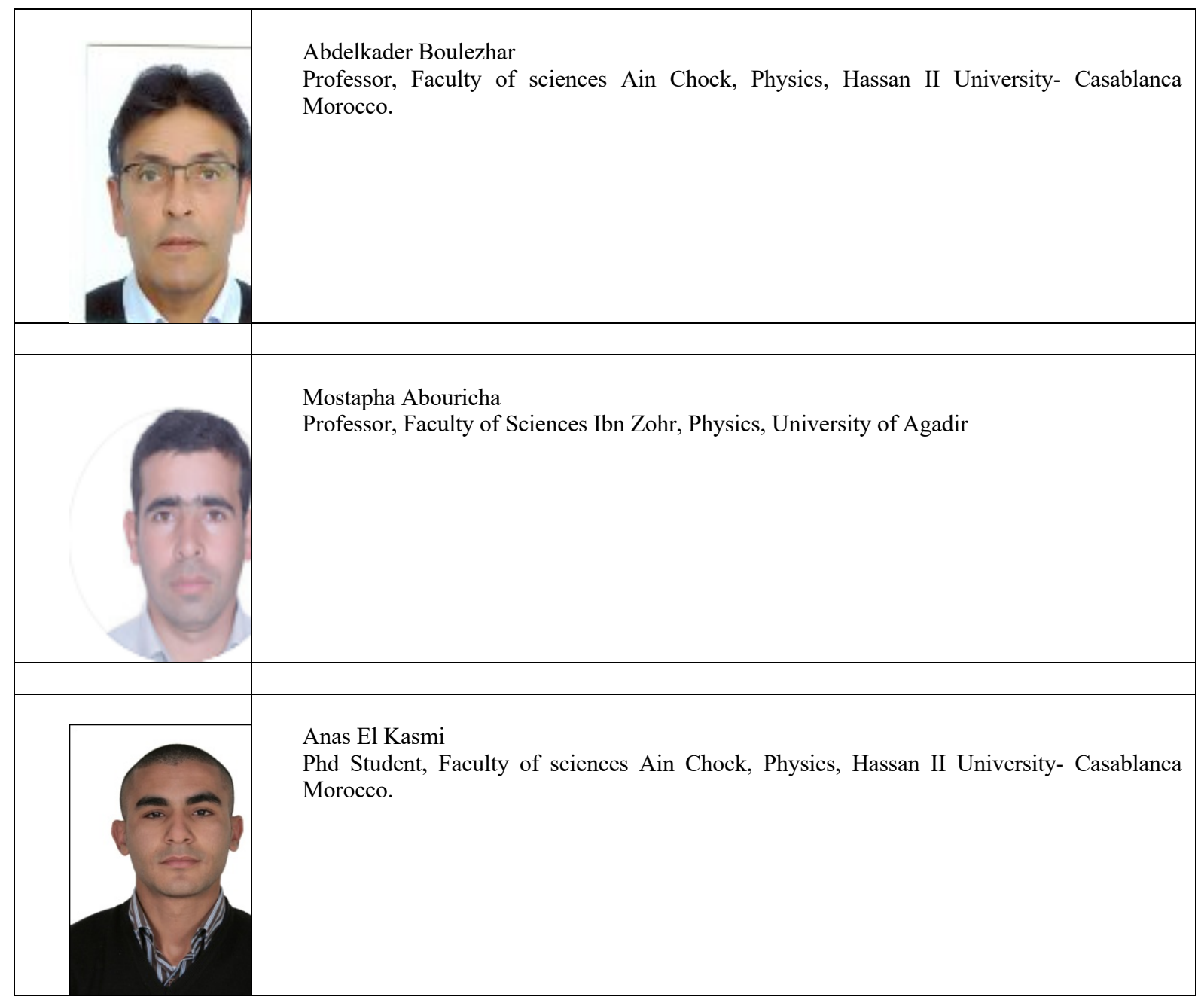

